\title{
Metodología de valoración de paisaje empleando ArcGIS: Aplicación a las Vías Pecuarias
}

\section{A landscape assessment methodology using ArcGIS: Application to traditional cattle migration routes}

$\underline{\text { A. Ezquerra }}^{(*)}$, M. Navarra ${ }^{(*)}$

\section{RESUMEN}

El concepto de paisaje cada vez adquiere mayor importancia en la legislación porque también es importante el estudio del paisaje y todas las herramientas que pueden ayudar a su valoración en la gestión de los recursos naturales, ya que dentro de la Ley de Patrimonio Natural y Biodiversidad, es necesario el estudio del paisaje en los Planes de Ordenación de los Recursos Naturales.

El avance de las técnicas que utilizan los Sistemas de Información Geográfica para la evaluación del paisaje permite agilizar su valoración con la introducción de numerosas variables que se incorporan en el análisis.

Este trabajo presenta una metodología de valoración del paisaje utilizando herramientas SIG, introduciendo variables y valorándolas en función de la cuenca visual y de la distancia al punto de observación, aplicándolo al caso particular de las Vías Pecuarias.

Palabras clave: Paisaje; métodos de valoración de paisaje; regresión múltiple; Sistemas de Información Geográfica.

\section{ABSTRACT}

The concept of landscape is assuming an increasing importance in legislation, and the Natural Heritage and Biodiversity Law requires a analysis of the landscape for the Natural Resources Ordination Plans. This calls for a range of tools to assist in studying the landscape prior to the assessment of management plans for natural resources.

The advances in the techniques employed by Geographic Information Systems for assessing the landscape can be enhanced by the incorporation of numerous variables into the analysis.

This work presents a landscape assessment methodology using GIS tools and including a number of variables which evaluate the landscape in terms of the viewshed and the distance from the observation point, applied to the specific case of the traditional cattle migration routes.

Keywords: Landscape; Geographical Information Systems; a landscape assessment methodology; multiple regression.

(*) Universidad Politécnica de Madrid (España).

Persona de contacto/Corresponding author: alejandra.ezquerra@upm.es (A. Ezquerra)

Cómo citar este artículo/Citation: Ezquerra, A., Navarra, M. (2015). Metodología de valoración de paisaje empleando ArcGIS: Aplicación a las Vías Pecuarias. Informes de la Construcción, 67(537): eo66, doi: http://dx.doi.org/10.3989/ic.13.051.

Licencia / License: Salvo indicación contraria, todos los contenidos de la edición electrónica de Informes de la Construcción se distribuyen bajo una licencia de uso y distribución Creative Commons Reconocimiento no Comercial 3.o. España (cc-by-nc). 


\section{INTRODUCCIÓN}

\subsection{Paisaje}

El creciente interés por la ecología y el medio ambiente se extiende también al paisaje (1) y para buscar su significado se han dado numerosas definiciones. A continuación se recopilan como punto de partida del trabajo.

Se define paisaje como la manifestación externa de los procesos que tienen lugar en el territorio, lo que significa una fuente de información que el hombre interpreta, analiza y experimenta (2).

Según Escribano (3) además de reconocer que se trata de una voz abstracta e intuitiva, paisaje se define con tres enfoques diferentes: artístico o estético, ecológico o geográfico y cultural:

- El enfoque artístico o estético se refiere a la combinación armoniosa de formas y colores.

- Según el enfoque ecológico o geográfico, se trata del estudio de las componentes naturales que lo forman.

- Por último el enfoque cultural hace alusión al escenario que supone el paisaje para la actividad humana.

Este autor también define tres componentes dentro del paisaje: uno formado por la porción de territorio, otro por la percepción de ese escenario y por último el hombre. Esto quiere decir que la interpretación personal causa un efecto en la interpretación del paisaje.

En esta línea, la Ley de Protección, Gestión y Ordenación del Paisaje de Cataluña entiende por paisaje, cualquier parte del territorio, tal y como la colectividad la percibe, cuyo carácter resulta de la acción de factores naturales o humanos y de sus interrelaciones (4). El paisaje (5) es, así, un segmento heterogéneo y dinámico de la naturaleza, reconocido por la conciencia de un individuo o una colectividad a través de sus sentidos, avalado por sus experiencias, sus conocimientos y el paradigma cultural imperante. Este segmento o la totalidad de un territorio con su respectiva estructura y función, es un producto de la compleja interrelación de los factores cualitativos y cuantitativos de los sistemas naturales y culturales, la cual actúa como un conjunto heterogéneo, dinámico e interdependiente, en un determinado espacio, tiempo y circunstancias históricas.

Ya en la década del año 2000, se introduce el concepto de paisaje cultural, sobretodo a raíz de del Consejo de Florencia. Paisaje cultural según Maldonado, L., 2011 (6) es aquel en el que se procura un análisis integrado de los distintos grupos de bienes que caracterizan e interactúan en las formas del hábitat y el aprovechamiento del medio natural. Se procura un análisis integrado de los distintos grupos de bienes que caracterizan e interactúan en las formas del hábitat y el aprovechamiento del medio natural.

Aguiló (7) recoge la definición de paisaje según dos conceptos Paisaje Ecológico y Paisaje Visual. Resume el paisaje como la combinación de la geomorfología, clima, plantas y animales y agua, y la incidencia de las acciones de tipo natural y antrópico. Esta definición da lugar al concepto de paisaje de dos formas diferentes según el tipo de estudio:

- La definición de paisaje ecológico sería «la consideración conjunta de componentes y procesos». En este sentido se distinguen dos sistemas, el fenosistema fácilmente perceptible, y el criptosistema o conjunto de procesos causales no fácilmente perceptibles que modifican el fenosistema.

- El paisaje visual o percibido es aquel en el que se tiene en cuenta al observador, tanto como sujeto de observación y como punto de observación. Por lo tanto se distinguen tantos paisajes como puntos de observación.

Otros autores definen los distintos tipos de paisaje desde una percepción más geográfica (8), atendiendo a la consideración de tres subsistemas:

- Abióticos: temperatura, humedad, rocas, suelo...

- Bióticos: flora, fauna.

- Antropizados: fruto de la actividad del hombre.

Al combinar los tres subsistemas se obtienen diferentes tipos de paisaje:

- Paisaje natural: estructura conformada por los subsistemas biótico y abiótico.

- Paisaje rural: donde se combinan los subsistemas biótico y abiótico con el antropizado.

- Paisaje urbano: donde predomina el subsistema antropizado junto con los subsistemas biótico y abiótico.

En este estudio se utilizará la definición más geográfica de paisaje, entendido como paisaje visual.

De este modo, se definen dos tipos de unidades de paisaje:

- Unidades homogéneas, que serían las que definen un mismo ecosistema.

- Unidades heterogéneas que definirían más de un ecosistema y que serían instrumentos denominados unidades de paisaje, que agruparían a porciones de territorio que responden uniformemente ante una acción exterior.

La noción de paisaje implica siempre la existencia de dos partes: aquella que es observada (el paisaje en sí) y aquella que observa y que vuelve a esa imagen algo existente. El paisaje puede ser muy variado dependiendo de un sinfín de elementos o situaciones tales como el momento del año en el que se observa, los elementos que lo componen o quizás hasta el punto de ubicación específico de aquel que observa los alrededores, punto de vista que es sin dudas subjetivo y que puede dar un significado completamente único y diferente a un mismo lugar.

Aquí es donde es importante señalar que el paisaje no debe ser nunca comprendido como una realidad estática (idea que puede generarse a partir de la representación gráfica del paisaje) si no como una realidad en permanente cambio y evolución. Esto es así no sólo debido a las fuerzas externas (como la acción del hombre) si no también a las fuerzas internas de los elementos que lo componen.

\subsection{Valoración de Paisaje}

Tomando como base la literatura existente, se puede afirmar que el paisaje está compuesto por descriptores y éstos se agrupan en componentes físicos (o elementos objetivos) y categorías estéticas (o elementos subjetivos).

Entre los componentes físicos (elementos objetivos) se consideran los siguientes: agua, forma del terreno, vegetación, 
nieve, fauna, usos del suelo, vistas, recursos culturales y elementos que alteran (Cañas, I., 1995) (9). Dentro de las categorías estéticas (elementos subjetivos) entrarían aquellos en que se tiene en cuenta la interpretación que de ellos hace el espectador. Existen diversos descriptores como el simbolismo, la unicidad, textura... (Cañas, I. 1995 y otros) (9). Para la valoración se consideran ambos incorporando un factor de ponderación.

Por otra parte, diversos autores (Fines, 1968, Garling, 1976, Craick, 1975, Gómez Orea, 1978, Stevenson, 1970; Hebblethwaite, 1973; Linton, 1968, Tandy, 1971; Ramos, 1980; Carlson, 1977, Dunn, 1974...) (7) han desarrollado métodos para la valorización del paisaje, que se deben tener también en cuenta por parte de los planificadores a la hora de gestionar el medio natural.

Aguiló (2001) (7) considera en la clasificación y estudio de métodos de valoración de calidad visual del paisaje, los estudios desarrollados por multitud de autores y organismos. Recoge la división de los métodos en directos, indirectos y mixtos.

Los métodos indirectos son aquellos que persiguen ser totalmente objetivos, los directos, asumen la subjetividad, y aquellos que son mixtos tienen en cuenta elementos objetivos y subjetivos. Los métodos objetivos, no tienen en cuenta la impresión que sobre paisaje tiene el observador, la valoración en este caso es de forma indirecta, es decir, sólo se cuantifica la valoración que los elementos objetivos tienen sobre paisaje. Los métodos subjetivos, al contrario, valoran solamente las impresiones que sobre paisaje tiene el espectador.

El método mixto trata de incorporar a la valoración objetiva la impresión del espectador, in situ ó mediante fotografías. Este método permite una respuesta no solamente indirecta, si no que tiene en cuenta la valoración del observador. Creando así metodologías, observación indirecta, que tienen en cuenta a los posibles usuarios finales, valoración directa.

\subsection{Evolución del concepto de Valoración de Paisaje}

Sobre la incorporación de técnicas para la valoración del paisaje y su caracterización, son pioneras las Agencias Americanas que comenzaron a estudiar su valoración entre finales de los años 60 y la década de los 70. En concreto el Forest Service dependiente del USDA, Bureau of Land Management (USDI) y el Soil Conservation Service (USDA).

Smardon (10) explica el desarrollo de los sistemas realizados por estas agencias. La aportación más importante de estos trabajos es su forma de evaluar los recursos estéticos, que se pueden resumir en:

- Forest Service: no daba demasiada importancia a los atributos estéticos y contiene como opinión de los observadores, la cantidad de personas que pueden ver el paisaje.

- Bureau of Land Management: establecía un término medio entre atributos físicos y estéticos y considera la cantidad de personas y la aptitud de éstas ante el paisaje

- Soil Conservation Service: consideraba los atributos físicos y daba especial importancia a los estéticos y tiene en cuenta: el número, frecuencia y duración de los observadores; tipo de observadores; localización y posición de observadores (11).
Es decir, estos métodos contienen también la valoración por parte de los observadores de las características del paisaje.

Cronológicamente, a partir de los años 80 se le ha otorgado especial importancia a los atributos estéticos. Se han empezado a emplear las encuestas para comparar las metodologías de evaluación de paisaje. Y ya en los 90, se valora no sólo la interpretación del elemento subjetivo por parte de los técnicos, si no que adquiere una importancia especial la opinión de los observadores que van a ser usuarios de los paisajes. En la década del 2000 - 2010 se ha seguido la misma tendencia, y además se ha resaltado la importancia de los recursos culturales.

\subsection{Regresión Múltiple}

Existen multitud de ejemplos que utilizan la Regresión Múltiple para valorar la calidad visual de los paisaje, como por ejemplo Arriaza et al. en 2005 que implementaron una metodología para valorar la calidad visual de los paisajes rurales y proponen aplicar la misma metodología en otras áreas para explicar su belleza escénica (12).

Ya desde 1969 Shafer et al. (1969) presentaron una relación entre métodos descriptivos y modelos de preferencia, denominado modelo integral. La aproximación se ha desarrollado en los últimos años y se sustenta en el uso de métodos estadísticos para determinar la relación entre las componentes del paisaje y las preferencias escénicas de los observadores (Palmer, 1983; Daniel and Vining, 1983; Buhyoff et al., 1994; Wherrett, 2000; Real et al., 2000; Daniel, 2001) (12).

En multitud de trabajos se ha desarrollado la valoración escénica de las preferencias de los observadores (Dunn, 1976; Law and Zube, 1983; Shafer and Brush, 1977; Shuttleworth, 1980b; Wherrett, 2000; Pérez, 2002) (12).

La Regresión Múltiple está representada por la siguiente fórmula:

$$
y=\sum_{i=1}^{n} b i . x i
$$

Siendo:

- $i=$ Número de variables objetivas.

- $b i=$ Coeficiente de ponderación donde se incluiría las categorías subjetivas.

$-x i=$ Componente objetiva.

Es a partir de la regresión múltiple, desde donde se ha calculado la fórmula de valoración de paisaje. Basándonos en la literatura existente, se ha determinado los recursos, y mediante una serie de encuestas, la componente objetiva, que convierte a cada uno de los recursos en atributos.

\subsection{Observadores y expertos}

La percepción y evaluación de los recursos estéticos del paisaje ha llegado a ser un área activa de interés en los últimos años. Existen dos aproximaciones diferentes en la valoración del paisaje, basado en juicios expertos y basados en la percepción -Lothian, 1999 (13)-. Mientras que los juicios expertos de la evaluación de los atributos estéticos son usados en la gestión de la naturaleza y el paisaje, los juicios de percepción, de observadores, son usados con propósitos científicos (Daniel, 2001) (13). 
Los juicios expertos están basados en la objetivación de los atributos estéticos (13) y se asumen idénticos a la opinión del público (Clay and Smidt, 2004) (13).

De acuerdo con Lothian (1999) (13), la mayor ventaja de los juicios expertos es su eficiencia práctica. Comparado con los juicios de observadores, los juicios expertos son más baratos y consumen menos tiempo.

Los juicios basados en la percepción, o de observadores, son una aproximación al elemento subjetivo. Están basados en la percepción subjetiva individual (13). Incluyen todos los aspectos de la relación del hombre con la naturaleza (Jessel, 2006) (13).

Una indiscutible ventaja de los juicios basados en la percepción, es el alto nivel de realidad incluso con pequeñas muestras de informadores (Daniel, 2001) (13).

Una desventaja en los juicios basados en la percepción, es que son relativamente caros, consumen mucho tiempo y son difíciles de organizar (Lothian, 1999) (13).

En el trabajo que se presenta se han utilizado los dos métodos:

- Juicio Experto, para la valoración de Corine, de la altitud del punto y de las pendientes.

- Juicio de Observadores, para calcular los coeficientes subjetivos de la Regresión Múltiple de la fórmula posteriormente calculada para valorar el paisaje.

\subsection{Nuevas herramientas GIS}

La aplicación de las tecnologías de información geográfica para analizar el paisaje es una de las tendencias punteras. En años pasados, se han realizado significantes avances utilizando los GIS en los ordenadores, lo que ha hecho posible el análisis de gran cantidad de información espacial (14).

En los pasados quince años, los avances en ordenadores y Sistemas de Información Geográfica (GIS) ha posibilitado el incremento del uso de estas tecnologías en la evaluación del paisaje visual (14).

Uno de estos ejemplos de cartografiar las cualidades visuales usando GIS fue utilizado por Steinitz (1990) (14). Utilizó GIS con la cobertura del terreno y el modelo digital del terreno del Acadia National Park, en USA, para cartografiar la calidad visual desde puntos de observación, con el comando viewshed, y empleó un análisis de regresión identificando ocho variables según las preferencias de los observadores (14).

Otro ejemplo es el trabajo llevado a cabo por el UK Living Landscaper Project. Desarrolló una estructura GIS basada en la caracterización del marco que describe el análisis del terreno (Warnock 2002; Countryside Agency (2004b) (14). El marco opera a diferentes niveles de resolución especial, desde el nivel regional (1:250.000), pasando por el nivel de país/distrito (1:50.000) hasta el nivel individual (1:10.000).

Lo que se pretende es tratar de difundir la importancia de este tipo de herramientas para los científicos medioambientales (Bishop, 2008) (15).
En este trabajo se ha tratado de idear un proceso, contrastado, para poder utilizar la herramienta informática ArcGIS, dado que permite el análisis de gran cantidad de cartografía, cada vez más abundante en la red.

\subsection{Fotografías}

Los medios fotográficos han sido utilizados para validar la representación del paisaje en los estudios de calidad visual del paisaje (Palmer and Hoffman 2001), y han estado muy extendidos (Law and Zube 1983; Palmer 2004; Pérez 2002; Arriaza et al. 2004) (14).

Estas fotografías son ofrecidas a los observadores para que emitan un juicio de valor del paisaje representado en ellas.

Las imágenes son utilizadas tanto en juicios expertos como en la valoración conforme al juicio de las personas (percepción).

En el trabajo que nos ocupa, se han realizado, en la primavera de 2011, una serie de fotografías, de una Cañada, situada en la pedanía del Cuadrón, Madrid.

\section{OBJETIVOS}

Los objetivos generales de este trabajo se pueden concretar en:

- Realizar un estudio del paisaje desde las vías pecuarias, incluyendo una nueva metodología de valoración del mismo, para poder conocer las posibilidades de estas vías.

- La aplicación de herramientas SIG, cada vez más potentes y con más información en la red (Directiva 2007/2, INSPIRE, horizonte 2011).

- Aproximarse a la utilización de un método de valoración de paisaje partiendo de la cartografía, de utilidad en la valoración del mismo en los Planes de ordenación de Recursos Naturales.

Para conseguirlos se ha seguido la siguiente metodología:

- Aplicar la fórmula calculada mediante encuestas, en la cual se obtiene el coeficiente subjetivo de ponderación de los diferentes recursos.

- Definir unos procesos en ArcGIS para calcular cada uno de los recursos que forman parte de la fórmula de la que se parte.

- Comprobar la viabilidad de esta primera aproximación con los resultados obtenidos en la Parte II de las encuestas.

- Realizar un análisis de resultados, comparando resoluciones de 100 y 200 metros.

\section{MATERIALES Y MÉTODOS}

\subsection{Observadores}

En este trabajo se han utilizado los dos métodos:

- Juicio Experto, para la valoración de Corine, de la altitud del punto y de las pendientes.

- Juicio de Observadores, para calcular los coeficientes subjetivos de la Regresión Múltiple de la fórmula posteriormente calculada para valorar el paisaje.

\subsection{Materiales}

Como objeto del trabajo se eligió una Vía Pecuaria, en concreto la «Cañada de Fuente Lagarto» situada en la pedanía del 
Cuadrón en la Comunidad de Madrid. A lo largo de 8 km se han tomado fotografías en 7 puntos diferentes, desde los cuatro puntos cardinales de cada punto seleccionado de esta infraestructura lineal; mostrándose como cuatripletas de cada punto y todos los puntos a la vez.

Se seleccionó un tramo de vía pecuaria a utilizar en el estudio, mediante tecnologías GIS y cartografía existente.

Para ello se procedió de la siguiente manera:

1. SELECCIÓN DE ZONAS DE ALTO VALOR PAISAJÍSTICO, cartografía del Atlas de los paisajes de España.

2. Selección de zONAS DE Alta CAlidAd, utilizando el Mapa de paisaje valorado para el uso recreativo en la CAM (Marta García García, 2004).

3. Superposición de las zonas SEleccionadas de ambos mapas.

- Determinación de los términos municipales incluidos en las áreas seleccionadas.

- Intersección mapa vías pecuarias (GCONA) x unidades alta calidad paisajística.

\subsection{Encuesta}

Para calcular la fórmula final de la Regresión Múltiple se realizaron encuestas entre enero y abril de 2012.

En total 205 fueron los encuestados. Como se trata de una distribución normal siguiendo el Modelo de Gauss, para la aplicación de esta herramienta es necesario realizar una encuesta lo suficientemente grande como para que permita cuantificar la subjetividad.

El tamaño de la muestra viene dado por la expresión:

$$
n=\left(\frac{\sigma\left(Z_{1-\alpha / 2}\right)}{d}\right)^{2}
$$

En nuestro caso el número necesario para un error (d) inferior al $10 \%$ y una varianza $(\sigma)$ de 0,5 sería de 97 encuestas.

$$
n=(0,5 \times 1,96 / 0,1)^{2}=96,4=97
$$

Como se ha comentado antes se realizaron 205 encuestas a distintos grupos de edad y formación, con una Parte I, y Parte II.

- En la Parte I, \% de influencia de cada una de las componentes.

- En la Parte II, valoración directa de 1 a 10 de las fotografías.

La encuesta es anónima y en ella se solicitaban datos del observador como: edad, nivel de estudios y sexo, para realizar posibles análisis posteriores.

\subsection{Información cartográfica}

Para la realización de este trabajo se ha empleado el paquete informático de información geográfica ArcGIS 10 de ESRI.

\subsubsection{Capas}

Las fuentes empleadas han sido las siguientes:
- Modelo digital del terreno (MDT). Se ha trabajado con dos tamaños de celda de 100 y 200 m metros. El MDT se ha obtenido de la página web del IGN. Y de aquí se obtiene información derivada como las cuencas visuales y la capa de pendientes.

- Bases cartográficas de la Comunidad de Madrid sobre municipios, vías pecuarias y pendientes. Esta información ha sido obtenida de www.madrid.org.

- Puntos de partida, obtenidos en campo sobre la vía pecuaria con el GPS. Se almacenaron tanto las coordenadas geográficas de los puntos como las cotas. Para poder exportar los datos del GPS a ArcGIS, se empleó el programa Trimble Office. Además las coordenadas tuvieron que ser previamente transformadas con Mercator y se les asignó el sistema de coordenadas UTM $50,30 \mathrm{~N}$.

- Corine Land Cover, 2006, uso 5. La información se ha obtenido del centro de descargas del Instituto Geográfico Nacional (IGN). La escala de Corine es de 1:100.000.

\subsection{Metodología}

\subsubsection{Regresión Múltiple}

La Regresión Múltiple sigue la siguiente ecuación:

$$
Y=y_{1}+y_{2}+\ldots+y_{n}
$$

Donde:

$$
\begin{aligned}
& y_{1}=b_{1} x_{1} . \\
& y_{2}=b_{2} x_{2} . \\
& \cdots \cdots \\
& y_{n}=b_{n} x_{n} .
\end{aligned}
$$

Con lo que:

$$
Y=b_{1} x_{1}+b_{2} x_{2}+\ldots+b_{n} x_{n}
$$

Donde $x_{i}$, son los atributos, y $b_{i}$ los coeficientes de la componente subjetiva calculada a partir de los observadores.

\subsubsection{Atributos}

Mediante consulta a la literatura científica existente sobre el tema se han seleccionado un conjunto de variables explicativas del paisaje:

- Factores Físicos: montañas, suelo, presencia de agua (16).

- Factores Biológicos: flora y fauna (16) (17).

- Recursos Culturales (9) (16) (18).

- Vistas (9).

- Elementos que alteran (7) (17).

De estos atributos, se pidió mediante encuestas que valoraran el tanto por ciento de intervención en la valoración de paisaje.

\subsubsection{Encuestas}

Las encuestas fueron realizadas entre enero y abril de 2012.

La encuesta es anónima y en ella se solicitaban datos del observador como: edad, nivel de estudios y sexo, para realizar posibles análisis. 
Constaba de dos partes:

Parte I

Se trataba de que el encuestado valorara en tanto por ciento el interés que para él representaban las variables obtenidas mediante el Muestreo Piloto. Es decir:

- Factores Físicos

- Factores Biológicos

- Vistas

- Recursos Culturales

- Elementos que alteran

Con ello se pretende medir la categoría de subjetividad que representan.

Ejemplo de esta parte de la encuesta en lo que se refiere a Factores Físicos aparece en la Tabla 1.

Parte II

En la Parte II: se mostraban una serie de fotografías en las que se solicitaba valorar de 1 a 10 la calidad del paisaje representado.

Esta valoración sirve para comprobar los resultados obtenidos en la FÓRMULA FINAL.

Como ejemplo para las fotografías tomadas desde los puntos 1,2 y 3 se puede observar en la Tabla 2.

En este estudio se emplearon fotografías para la representación del paisaje. Las fotografías fueron tomadas desde siete puntos de observación, elegidos aleatoriamente, en una infraestructura lineal de fácil acceso. Fueron tomadas según las cuatro direcciones cardinales de cada punto.

La serie de fotografías fue realizada por los autores en la primavera de 2011 (Figuras 1, 2, 3, 4, 5, 6, 7), en 7 puntos distintos.

Como se ha señalado, el trabajo se realiza en un tramo de la Cañada de Fuente Lagarto, a su paso por los municipios de la Sierra Norte de la Comunidad de Madrid de Garganta de los Montes, Lozoyuela-Navas-Sieteiglesias, y que parte de la pedanía de El Cuadrón.

Se tomaron cuatro fotografías de cada punto, de frente, atrás, izquierda y derecha.

El resultado de los siete puntos es el siguiente:

- Grupo de fotografías desde el punto 1 (Figura 1).

- Grupo de fotografías desde el punto 2 (Figura 2).

- Grupo de fotografías desde el punto 3 (Figura 3).

- Grupo de fotografías desde el punto 4 (Figura 4).

- Grupo de fotografías desde el punto 5 (Figura 5).

- Grupo de fotografías desde el punto 6 (Figura 6).

- Grupo de fotografías desde el punto 7 (Figura 7).
Tabla 1. Encuesta Parte I.

\begin{tabular}{|l|l|l|l|}
\hline FACTORES FÍSICOS: & & & \% \\
\hline & Forma del terreno & $\%$ & \\
\hline & & Llano & \\
\hline & & Colina & \\
\hline & & Montaña & \\
\hline & & TOTAL & 100 \\
\hline & Nieve & $\%$ & \\
\hline & Usos del suelo & $\%$ & \\
\hline & Agua & $\%$ & \\
\hline & TOTAL & 100 & \\
\hline & & & \\
\hline
\end{tabular}

Tabla 2. Encuesta Parte II.

\begin{tabular}{|c|c|c|}
\hline \multicolumn{2}{|r|}{ PUNTO } & Calificación \\
\hline \multirow{4}{*}{$\mathbf{1}$} & FRENTE & \\
\hline & ATRÁS & \\
\hline & DERECHA & \\
\hline & IZQUIERDA & \\
\hline \multirow{4}{*}{2} & FRENTE & \\
\hline & ATRÁS & \\
\hline & DERECHA & \\
\hline & IZQUIERDA & \\
\hline \multirow{4}{*}{3} & FRENTE & \\
\hline & ATRÁS & \\
\hline & DERECHA & \\
\hline & IZQUIERDA & \\
\hline
\end{tabular}

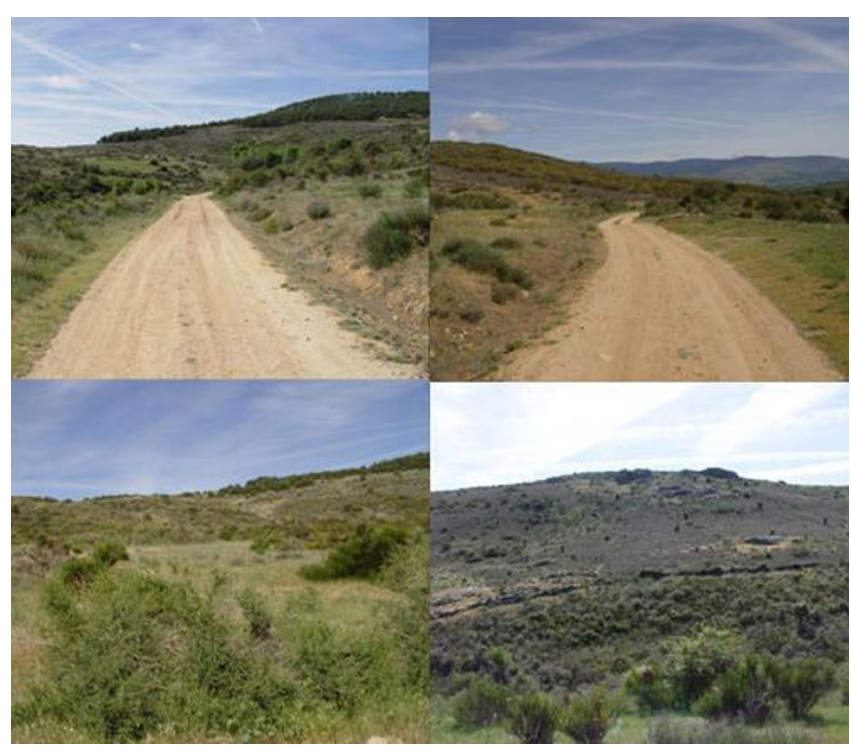

Figura 1. Grupo de fotografías desde el punto 1. 


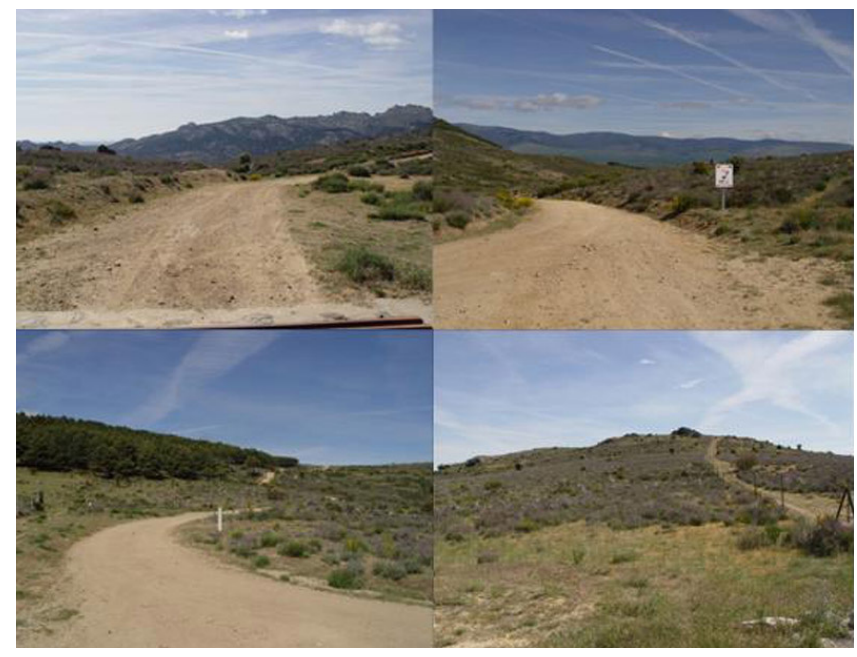

Figura 2. Grupo de fotografías desde el punto 2.

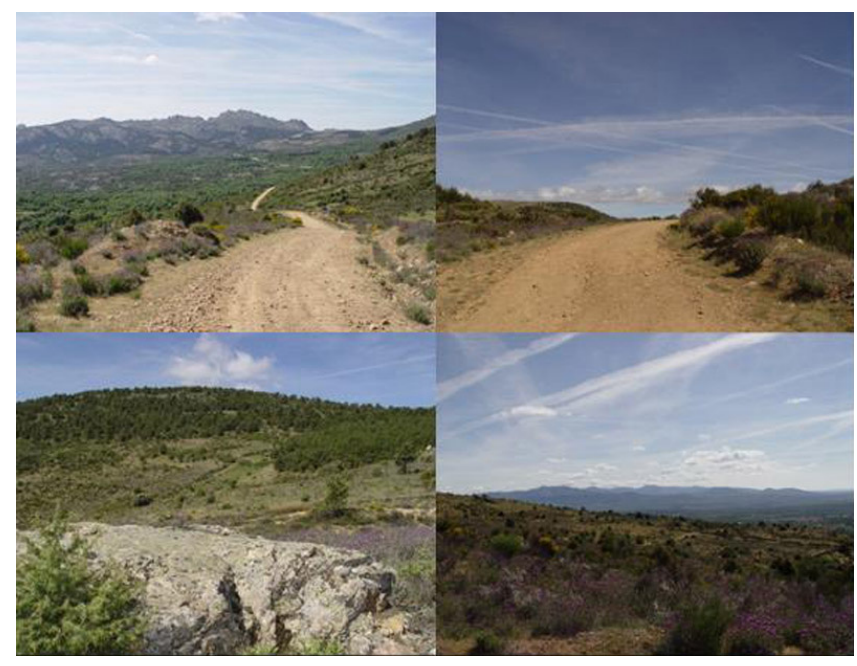

Figura 3. Grupo de fotografías desde el punto 3.

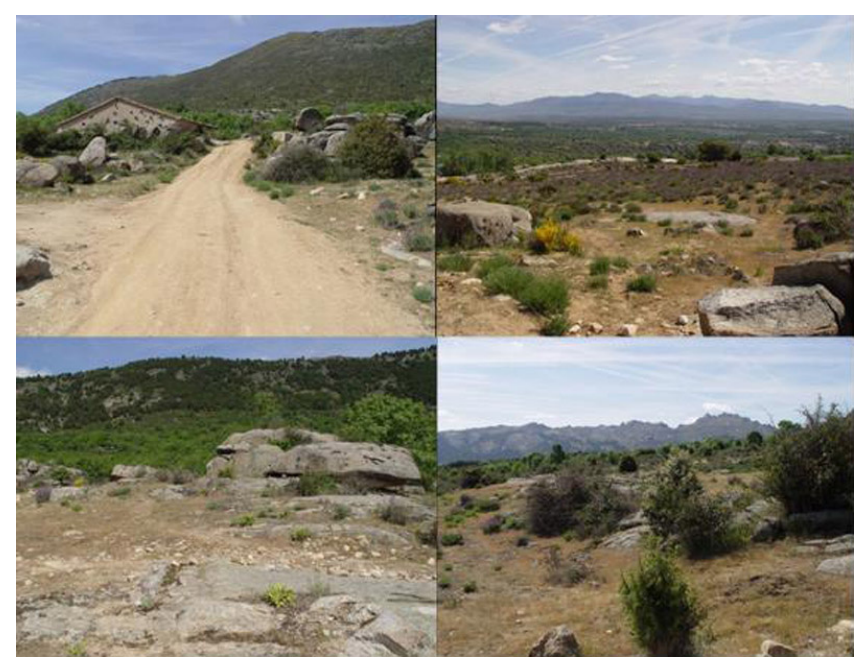

Figura 4. Grupo de fotografías desde el punto 4.

\subsection{Variables}

\subsubsection{Recursos Físicos}

Para la valoración de los recursos físicos en el SIG se aplica el siguiente modelo.

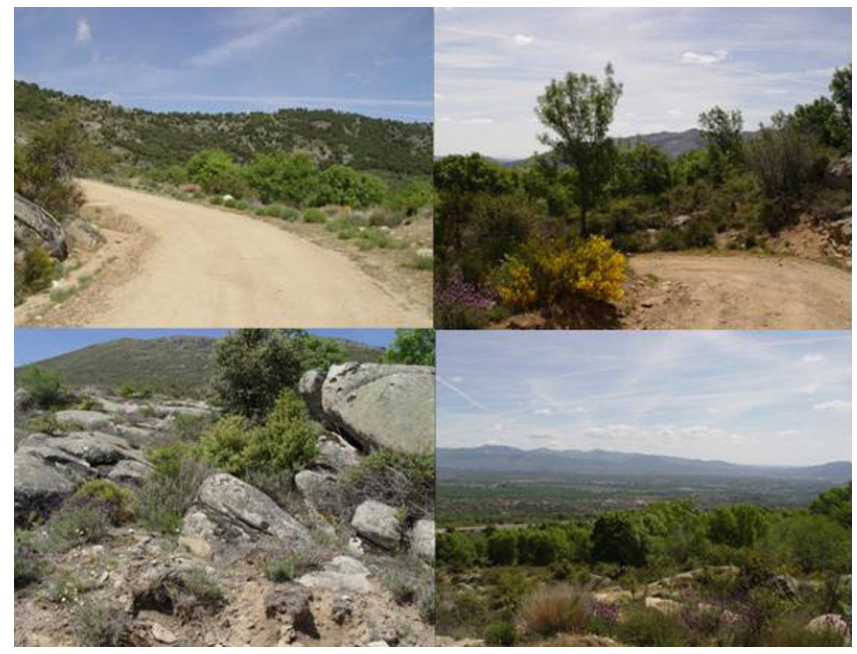

Figura 5. Grupo de fotografías desde el punto 5.

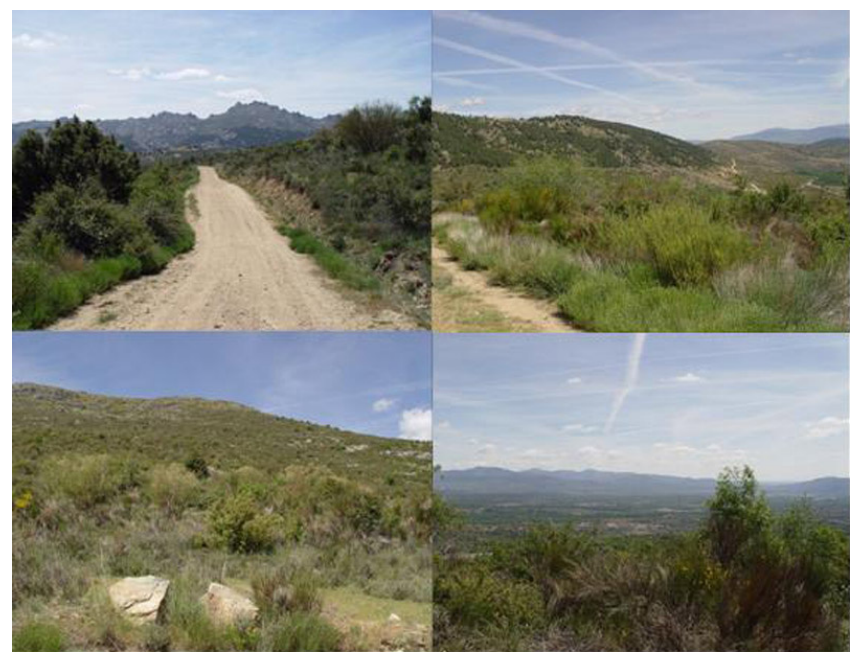

Figura 6. Grupo de fotografías desde el punto 6.

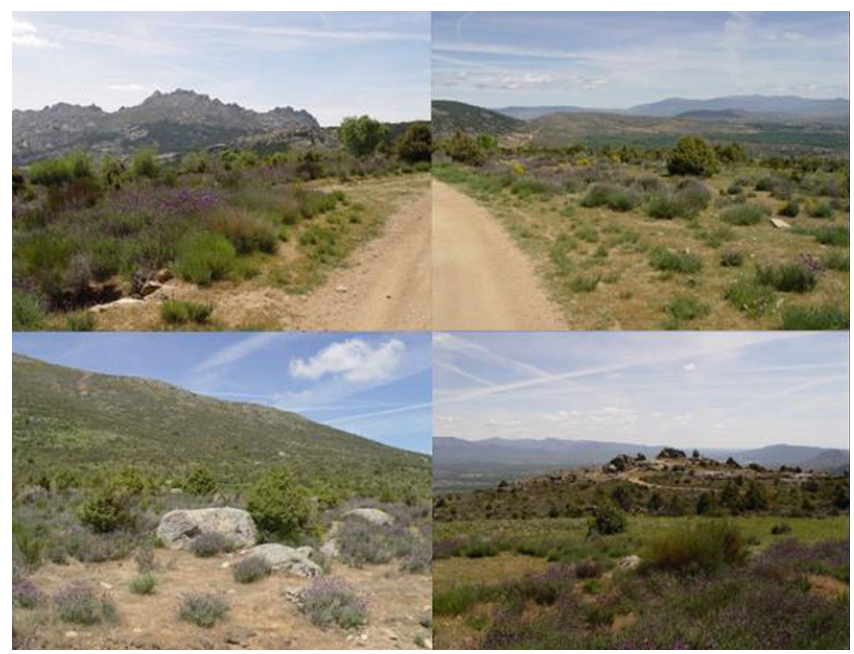

Figura 7. Grupo de fotografías desde el punto 7.

$F \cos =(1 / 2 \cdot$ Valor Paisaje de Altitud del punto + $+1 / 2$ Valor Paisaje de las Pendientes de la Cuenca)

Los recursos físicos serán ponderados con el coeficiente 0,35 según la ecuación de valoración del paisaje. 


\subsubsection{Valor de Paisaje según la altitud del punto}

Para los recursos físicos se parte de las alturas de los puntos, tomadas mediante GPS, y de las pendientes circundantes.

Para valorar la influencia de la altitud, se utiliza la valoración de Cañas (1995) (9), representado en la Tabla 3.

Tabla 3. Valor de Paisaje según la Altitud.

\begin{tabular}{|l|c|}
\hline Altitud & Valor de Paisaje \\
\hline Llano (menos de 100 m) & o \\
\hline Colina (o a 100 m) & 2 \\
\hline Costa & 6 \\
\hline Alturas de 100 a 700 Cerros & 6 \\
\hline Montaña, Superior a 700 m & 8 \\
\hline
\end{tabular}

\subsubsection{Valor de Paisaje según las pendientes de la cuenca}

Para la valoración de las pendientes se calcula en primer lugar cuál sería el valor de la cuenca si todo el entorno, en un determinado radio de distancia (en este caso 5.000 metros), fuera visible.

En la Tabla 4 se muestra la valoración del paisaje en función de las pendientes.

Tabla 4. Valor de Paisaje según las Pendientes.

\begin{tabular}{|l|c|}
\hline Tipo de Pendiente & Valor de Paisaje \\
\hline Del 0\% al 3\% & 2 \\
\hline Del 3\% al 12\% & 4 \\
\hline Del 12\% al 20\% & 6 \\
\hline Del 20\% al 35\% & 8 \\
\hline Más del 35\% & 10 \\
\hline Pendientes sin clasificar & 0 \\
\hline
\end{tabular}

\subsubsection{Recursos Biológicos}

Se realizan dos valoraciones, una según el recurso biológico en el punto de observación (Valor de Paisaje del punto) y otra del valor de paisaje de la vegetación en la cuenca visual. Una vez obtenidos son ponderados por su coeficiente en la ecuación [1].

El resultado sería:

$$
0,36 \times \text { Corine }+0,36 \times \text { Corine } \times \text { Viewshed } \times 1 / d
$$

(Corine: Valor de uso de suelo según el Corine, Coordination of Information on the Enviromental, Land Cover).

\subsubsection{Valor de Paisaje según la vegetación del punto}

Para obtener el valor en el punto de observación se parte de la siguiente valoración (Tabla 5) de los usos en el Corine (uso 5).

Se asigna un valor a cada punto, y se multiplica por el factor de ponderación, que en este caso es o,36 (obtenida de la importancia que los encuestados dieron a este parámetro).
Tabla 5. Valor de Paisaje según los Usos Corine.

\begin{tabular}{|c|c|}
\hline Uso & Valor de Paisaje \\
\hline Otros pastizales mediterráneos & 6 \\
\hline Embalses & 10 \\
\hline Matorral boscoso de bosque mixto & 6 \\
\hline Tejido urbano continuo & 1 \\
\hline Matorral boscoso de frondosas & 7 \\
\hline $\begin{array}{l}\text { Matorrales subarbustivos o arbustivos muy } \\
\text { poco densos }\end{array}$ & 7 \\
\hline Matorral boscoso de frondosas & 7 \\
\hline Urbanizaciones exentas y/o ajardinadas & 1 \\
\hline Matorral boscoso de frondosas & 7 \\
\hline $\begin{array}{l}\text { Pastizales prados o praderas con arbolado } \\
\text { adehesado }\end{array}$ & 6 \\
\hline $\begin{array}{l}\text { Grandes formaciones de matorral denso o } \\
\text { medianamente denso }\end{array}$ & 7 \\
\hline Matorral boscoso de frondosas & 7 \\
\hline Urbanizaciones exentas y/o ajardinadas & 1 \\
\hline Matorral boscoso de frondosas & 7 \\
\hline Urbanizaciones exentas y/o ajardinadas & 1 \\
\hline $\begin{array}{l}\text { Grandes formaciones de matorral denso o } \\
\text { medianamente denso }\end{array}$ & 7 \\
\hline Matorral boscoso de bosque mixto & 7 \\
\hline Caducifolias y marcescentes & 8 \\
\hline $\begin{array}{l}\text { Pastizales prados o praderas con arbolado } \\
\text { adehesado }\end{array}$ & 6 \\
\hline Praderas & 6 \\
\hline Tejido urbano continuo & 1 \\
\hline Urbanizaciones exentas y/o ajardinadas & 1 \\
\hline Matorral boscoso de frondosas & 7 \\
\hline Matorral boscoso de coníferas & 7 \\
\hline $\begin{array}{l}\text { Matorrales subarbustivos o arbustivos muy } \\
\text { poco densos }\end{array}$ & 7 \\
\hline $\begin{array}{l}\text { Pastizales prados o praderas con arbolado } \\
\text { adehesado }\end{array}$ & 7 \\
\hline $\begin{array}{l}\text { Grandes formaciones de matorral denso o } \\
\text { medianamente denso }\end{array}$ & 7 \\
\hline $\begin{array}{l}\text { Matorrales subarbustivos o arbustivos muy } \\
\text { poco densos }\end{array}$ & 6 \\
\hline $\begin{array}{l}\text { Matorrales subarbustivos o arbustivos muy } \\
\text { poco densos }\end{array}$ & 6 \\
\hline Bosques de coníferas con hojas aciculares & 9 \\
\hline Pastizales supraforestales mediterráneos & 6 \\
\hline Afloramientos rocosos y canchales & 8 \\
\hline
\end{tabular}

\subsubsection{Valor de Paisaje según la vegetación de la cuenca visual}

El primer paso es calcular la cuenca visual desde el punto, utilizando el MDT. Utilizando el software ArcGis, se define un Extent de $3.000 \mathrm{~m}$ a cada coordenada del punto con el objeto de limitar el cálculo.

Según los modelos habituales utilizados en el estudio de la visibilidad (método Run Visual Range, utilizado, por ejemplo, en los aeropuertos para medir la visibilidad de los pilotos), la 
calidad visual decrece según el inverso de la distancia hasta $3.000 \mathrm{~m}$, y de 3.000 a $5.000 \mathrm{~m}$ de forma logarítmica. De esta forma, para valorar ese descenso de la calidad visual se calcula el inverso de la distancia a $3.000 \mathrm{~m}$, despreciándose el decrecimiento logarítmico por considerarse prácticamente nulo.

\subsubsection{Vistas}

Intuitivamente, una mayor amplitud, supone un mayor valor para el paisaje, por tanto, la metodología empleada parte de la búsqueda del número de celdas que se ven en una distancia euclidiana de $5.000 \mathrm{~m}$, umbral de la visión, sin tener en cuenta el MDT., el máximo.

Se calcula el número de celdas. En el caso de una resolución de las celdas de $100 \mathrm{~m}$, el total de celdas sería de 7.793.

Con este dato se divide en 10 intervalos, para crear una tabla (Tabla 6) que nos permita calcular el valor de paisaje de cada punto. Teniendo en cuenta que a mayor número de celdas, mayor valor de paisaje.

Tabla 6. Valor de Paisaje según las Vistas.

\begin{tabular}{|c|c|}
\hline Número de Celdas & Valor \\
\hline $0-780$ & 1 \\
\hline $780-1560$ & 2 \\
\hline $1560-2340$ & 3 \\
\hline $2340-3120$ & 4 \\
\hline $3120-3900$ & 5 \\
\hline $3900-4680$ & 6 \\
\hline $4680-5460$ & 7 \\
\hline $5460-6240$ & 8 \\
\hline $6240-7020$ & 9 \\
\hline $7020-7800$ & 10 \\
\hline
\end{tabular}

Posteriormente se calcularían las cuencas visuales, basándonos en el MDT.

Introduciéndonos en la tabla se obtendría el valor de las vistas, según el número de celdas que se ven en una distancia euclideana de $5.000 \mathrm{~m}$, teniendo en cuenta el MDT.

\subsubsection{Recursos Culturales}

Para la objetivación de este elemento subjetivo, en la ecuación de valoración del paisaje se aplica el coeficiente de 0,11, que es la importancia que los encuestados dieron a este parámetro.

La Tabla 7 se obtiene de la valoración de juicio experto.

Tabla 7. Tabla de Valor de Paisaje según los Recursos Culturales.

\begin{tabular}{|l|c|}
\hline $\begin{array}{c}\text { Valor de Paisaje según } \\
\text { Recursos Culturales }\end{array}$ & Valor \\
\hline Ninguno & 0 \\
\hline Bajo & $1-3$ \\
\hline Medio & $4-7$ \\
\hline Alto & $7-10$ \\
\hline
\end{tabular}

\subsubsection{Elementos que alteran}

La objetivación de este parámetro es de -0,11 que es la importancia que los encuestados dieron a este parámetro.

Según la valoración de los juicios expertos el Valor de Paisaje que adquieren se resume en la Tabla 8.

Tabla 8. Valor de Paisaje según los Elementos que alteran.

\begin{tabular}{|l|c|}
\hline \multicolumn{1}{|c|}{$\begin{array}{c}\text { Grado de intrusión de los } \\
\text { Elementos que alteran }\end{array}$} & $\begin{array}{c}\text { Valor de } \\
\text { Paisaje }\end{array}$ \\
\hline Ninguno & 0 \\
\hline Bajo & $1-3$ \\
\hline Medio & $4-7$ \\
\hline Alto & $8-10$ \\
\hline
\end{tabular}

\section{RESULTADOS Y CONCLUSIONES}

\subsection{Resultados de las encuestas}

El resultado final es la obtención de una metodología para valorar el paisaje desde infraestructuras lineales, teniendo en cuenta componentes y categorías. Es decir, existe una relación entre $x_{i}$ e $y_{i j}$ que se pueden aproximar a una recta de pendiente $b_{i}$, siendo $i$ de 1 a 5 :

$i=1$ : Factores Físicos, $b_{1}=0,35$

$i=2$ : Factores Biológicos, $b_{2}=0,36$

$i=3$ : Vistas, $b_{3}=0,18$

$i=4$ : Recursos Culturales, $b_{4}=0,11$

$i=5$ : Elementos que alteran, $b_{5}=0,11$

Datos procedentes de la media de las encuestas realizadas, tomando como error inferior al $10 \%$ y avalado además por la varianza que es menor de 0,5 , en cada uno de los casos.

\subsection{Resultado de la fórmula}

La FÓRMULA FINAL, expresada en tanto por uno:

$\mathrm{V}=0,35 \mathrm{~F} \cos +0,36 \mathrm{~B} \cos +0,18 \mathrm{Vis}+0,11 \mathrm{RC}-0,11 \mathrm{EA}$

Donde:

- V: Valor total. Puntúa de o a 10.

- Fcos, X1: Elementos físicos: forma del terreno, presencia de agua o nieve. Varía entre 10, 5, o

- Bcos, X2: Elementos Biológicos: presencia de fauna y flora. Varía entre o, 5 y 10.

- Vis, $\mathrm{X}_{3}$ : Vistas. Varían entre o, 5 y 10.

- RC, X4: Recursos Culturales. Varían entre o, 5 y 10.

- EA, X5: Elementos que alteran. Presencia de cables eléctricos, edificaciones. Varían entre o, 5 y 10.

\subsection{Conclusiones del método mixto}

Los resultados de la encuesta han sido contrastados con una valoración independiente mediante un Método Directo. Los resultados, que sirven para contrastar, avalan la FÓRMULA FINAL en una valoración total de todos los PUNTOS. Al comprobar la varianza $<$ de 0,5 , y el error $<0,1$, se han cumplido objetivos. Sin embargo, un error menor podría conseguirse aumentando el tamaño de la muestra de la encuesta. 


\subsection{Resultado ArcGIS}

Una vez obtenidas en el SIG las distintas variables intermedias a considerar en la ecuación de valoración del paisaje es posible obtener el valor final para cada uno de los puntos. En este apartado se presentan los resultados finales y la comparación con las valoraciones obtenidas a partir de la encuesta. Con el fin de testar la metodología usada en el SIG se ha calculado la diferencia entre el valor obtenido en el SIG y en la encuesta. Finalmente, los resultados se presentan para los cálculos realizados con una resolución de celdas de 100 y otras de 200 metros.

\section{A) Tamaño de celda 100}

En la Tabla 9 se presentan los resultados aplicando la metodología SIG completa (valoran las pendientes de la Cuenca Visual) y con un tamaño de celda de 100 metros. Como puede verse las diferencias con los resultados obtenidos en las encuestas son muy pequeñas y más o menos homogéneas entre todos los puntos.

\section{B) Tamaño de celda 200}

Otras de las posibilidades que se han considerado, es tomar un tamaño de celda diferente (en este caso 200 metros) y ver cómo este influye el tamaño de la unidad espacial en la valoración del paisaje. Los resultados obtenidos (Tabla 10) muestran la importancia del tamaño de celda a considerar, al producirse una diferencia mucho mayor con respecto a los valores encontrados en las encuestas.

\subsection{Conclusiones para tamaño de celda 100 y para tamaño de celda 200}

Una de las preguntas que se realizan los planificadores del territorio es cómo los SIG pueden ser mejorados para analizar la calidad del paisaje (19). Con este método se ha tratado de investigar sobre esta cuestión, en varios sentidos, obteniendo las siguientes conclusiones:

- Se ha elaborado una metodología que se adapta bien a un SIG. Esto nos ha permitido agilizar los análisis, tanto en tiempo y coste, frente a métodos como la realización de encuestas a partir de fotografías, y mejorar los resultados frente a otros análisis. Así, se ha podido ver como los resultados obtenidos en el SIG son similares a los obtenidos mediante encuestas con fotografías, con la ventaja de que, una vez desarrollada y testada la metodología, ésta es fácilmente aplicable a todo un recorrido lineal, frente al trabajo que supondría tomar fotos a lo largo de un recorrido largo y el costo que supone la realización de una encuesta amplia. A la vez, se ha mostrado como la agilidad de los SIG permite introducir numerosas variables de entorno y valorarlas en función de la cuenca visual y de la distancia al punto de observación, lo que mejora los resultados sobre metodologías más sencillas, que sólo consideran el valor de determinadas variables en el punto (tal como hace Cañas I (1995) (9) con la altura del punto).

- En este trabajo se han mejorado esas metodologías en dos elementos. Por un lado se han establecido unas cuencas de 5.000 para Vistas y Recursos Físicos y 3.000 m para Recursos Biológicos. Además, debido a que estos últimos implican una apreciación de la unidad de visión y esta decrece con la distancia, las variables de entorno se han ponderado según una caída lineal con la distancia en los primeros 3.000 metros. Por otro, frente a la metodología de Cañas (1995) (9) en la valoración de los recursos físicos se han considerado el estudio de las pendientes y la altura. Otros puntos que deberían estudiarse más ampliamente.

- Se ha estudiado el efecto del diferente tamaño de la unidad espacial en los resultados (problema de la unidad espacial modificable, la UEM), viendo como la metodología usadas

Tabla 9. Resultados con tamaño de celda 100.

\begin{tabular}{|l|c|c|c|c|c|c|c|c|c|}
\hline & \multicolumn{7}{|c|}{ Resultados usando metodología en ArcGIS } & \multicolumn{2}{c|}{ Resultados usando encuestas/fotos } \\
\hline & Rfcos & Rbcos & RBAlrededor & Vistas & RC & EA & Total & Valor Moda & Valor Media \\
\hline Punto 1 & 1,48 & 2,16 & 0,8 & 0,18 & 0,55 & 0 & 5,17 & 6 & 5,65 \\
\hline Punto 2 & 1,60 & 2,16 & 0,56 & 0,18 & 0,55 & 0 & 5,05 & 5,25 & 4,76 \\
\hline Punto 3 & 1,82 & 2,16 & 1 & 0,54 & 0,55 & 0 & 6,07 & 6,5 & 6,51 \\
\hline Punto 4 & 1,71 & 2,16 & 1,17 & 0,54 & 0,55 & 0 & 6,13 & 6,5 & 6,25 \\
\hline Punto 5 & 1,78 & 2,16 & 1,5 & 0,54 & 0,77 & 0 & 6,75 & 7 & 6,64 \\
\hline Punto 6 & 1,84 & 2,16 & 1,73 & 0,54 & 0,55 & 0 & 6,82 & 6,25 & 5,95 \\
\hline Punto 7 & 1,66 & 2,52 & 1,29 & 0,54 & 0,55 & 0 & 6,56 & 6,75 & 6,55 \\
\hline \multicolumn{78}{|c|}{ MEDIAS } & 6,08 & 6,32 & 6,04 \\
\hline
\end{tabular}

Tabla 10. Resultados con tamaño de celda 200.

\begin{tabular}{|l|c|c|c|c|c|c|c|c|c|}
\hline & \multicolumn{7}{|c|}{ Resultados usando metodología en ArcGIS } & \multicolumn{2}{c|}{ Resultados usando encuestas/fotos } \\
\hline & 1/2Rfcos & Rbcos & Alrededor & Vistas & RC & EA & TOTAL & Valor Moda & Valor Media \\
\hline Punto 1 & 1,46 & 2,16 & 0,2 & 0,18 & 0,55 & 0 & 4,55 & 6 & 5,65 \\
\hline Punto 2 & 1,69 & 2,16 & 0,27 & 0,36 & 0,55 & 0 & 5,03 & 5,25 & 4,76 \\
\hline Punto 3 & 1,46 & 2,16 & 0,28 & 0,54 & 0,55 & 0 & 4,99 & 6,5 & 6,51 \\
\hline Punto 4 & 1,84 & 2,16 & 0,42 & 0,54 & 0,55 & 0 & 5,51 & 6,5 & 6,25 \\
\hline Punto 5 & 1,68 & 2,16 & 0,46 & 0,36 & 0,77 & 0 & 5,43 & 7 & 6,64 \\
\hline Punto 6 & 1,87 & 2,16 & 0,49 & 0,72 & 0,55 & 0 & 5,79 & 6,25 & 5,95 \\
\hline Punto 7 & 1,68 & 2,52 & 0,31 & 0,36 & 0,55 & 0 & 5,42 & 6,75 & 6,55 \\
\hline
\end{tabular}


es muy sensible a este problema. Los mejores resultados se obtienen con un tamaño $100 \mathrm{~m}$, con diferencias importantes sobre los $200 \mathrm{~m}$. Esto supone la necesidad de considerar este problema y testar los resultados cuando la metodología se aplique en otros espacios.

El estudio del paisaje es importante para que no decaiga el uso de estas vías que han perdido su utilización tradi- cional, dentro de los programas de Desarrollo Rural de la UE.

También es importante el estudio del paisaje y todas las herramientas que pueden ayudar a ello en la gestión de los recursos naturales, ya que dentro de la Ley de Patrimonio Natural y Biodiversidad, es necesario el estudio del paisaje en los Planes de Ordenación de los Recursos Naturales.

\section{REFERENCIAS}

(1) Otero, I., Varela, E., Mancebo, S., Ezquerra, A. (2009). Análisis de visibilidad en la evaluación de impacto ambiental de nuevas construcciones. Informes de la Construcción, 61(515): 67-75, doi: http://dx.doi.org/10.3989/ic.09.014.

(2) Nila de Taranco. (2005). www.fundicot.org.

(3) Escribano-Bombín, M. ${ }^{a}$ M. (1987) El Paisaje. Editorial MOPU.

(4) Generalitat de Catalunya. (2005, 8 de julio). Ley 8/2005, de 8 de junio de 2005, de protección, gestión y ordenación del paisaje. Boletín Oficial del Estado, ${ }^{0}{ }^{162}$. España.

(5) Centro Argentino de Arquitectos Paisajistas. www.caapaisajistas.org.ar.

(6) Maldonado, L., Vela-Cossío, F. (2011). El patrimonio arquitectónico construido con tierra. Las aportaciones historiográficas y el reconocimiento de sus valores en el contexto de la arquitectura popular española. Informes de la Construcción, 63(523): 71-80, doi: http://dx.doi.org/10.3989/ic.10.062.

(7) Aguiló-Alonso, M. (2001). Guía Metodológica para Estudios del Medio Físico. Madrid: Ministerio de Medio Ambiente.

(8) Navés-Viñas, F., Arosemena-Díaz, G. (2005). Arquitectura del paisaje rural de la Península Ibérica, Islas Baleares y Canarias. Barcelona: Editorial Omega.

(9) Cañas, I. (1.995). Valoración del Paisaje. Lugo: Unicopia.

(10) Smardon, R.C. (1986). Historical Evolution of Visual Resource Management within Three Federal Agencies. Journal Environment Management, 22: 301-317.

(11) Cañas, I. (1995). Introducción al Paisaje. Lugo: Unicopia.

(12) Arriaza, M., Cañas, J. F., Cañas, J. A., Ruiz, P., González, J., Barea, F. (2005, 24-27 de agosto). Landscape assessment via regression analysis. En XIth Congress of the EAAE (European Association of Agricultural Economists). Copenhagen, Denmark.

(13) Skrivanova, Z., Kalivoda, O. (2010). Perception and assessment of landscape aesthetic values in the Czech Republic - a literature review. Journal of Landscape Studies, 3: 211-220.

(14) Wu, Y., Bishop, I., Hossain, H., Sposito, V. (2006). Using SIG in landscape visual quality assessment. Applied Gis, 2(3).

(15) Bishop, I., (2008). Integration of augmented reality and GIS: A new approach to realistic landscape visualisation. Landscape and Urban Planning, 86(3-4): 226-232, doi: http://dx.doi.org/10.1016/j.landurbplan.2008.03.004.

(16) E. Pavlikakis, G., A. Tsihrintzis, V. (2005). Perceptions and preferences of local population in Eastern Macedonia and Thrace National Park in Greece. Landscape and Urban Planning, 77(1-2): 1-16, doi: http://dx.doi.org/10.1016/j.landurbplan.2004.12.008.

(17) Rogge, E., Nevens, F., Gulinck, H. (2007). Perception of rural landscapes in Flanders: Looking beyond aesthetics. Landscape and Urban Planning, 82(4): 159-174, doi: http://dx.doi.org/10.1016/j.landurbplan.2007.02.006.

(18) Stephenson, J. (2008) The Cultural values model: An integrated approach to values. Landscape and Urban Planning, 84(2): 127-139, doi: http://dx.doi.org/10.1016/j.landurbplan.2007.07.003.

(19) J. Pettit, C., M. Raymond, C., A. Bryan, B., Hayden, L. (2011). Identifying streghs and weaknesses of landscape visualization for effective communication of future alternatives. Landscape and Urban Planning, 100(3): 231-241, doi: http:// dx.doi.org/10.1016/j.landurbplan.2011.01.001. 